\title{
Erratum: Origin of strong dispersion in Hubbard insulators [Phys. Rev. B 92, 075119 (2015)]
}

\author{
Y. Wang, K. Wohlfeld, B. Moritz, C. J. Jia, M. van Veenendaal, K. Wu, C.-C. Chen, and T. P. Devereaux
}

Q (Received 19 May 2018; published 29 May 2018)

DOI: 10.1103/PhysRevB.97.199903

There is an incorrect sign in the definition of the Hamiltonian terms depicting the three-site terms $\left(\mathcal{H}_{3 s}\right)$ in the third line of Eq. (2). The correct equation is

$$
\mathcal{H}_{3 s}=-\frac{J_{3 s}}{4} \sum_{\substack{\langle\mathbf{i} \mathbf{j}\rangle,\left\langle\mathbf{i} \mathbf{j}^{\prime}\right\rangle \\ \mathbf{j} \neq \mathbf{j}^{\prime}, \sigma}}\left(\tilde{c}_{\mathbf{j}^{\prime} \sigma}^{\dagger} \tilde{n}_{\mathbf{i} \bar{\sigma}} \tilde{c}_{\mathbf{j} \sigma}-\tilde{c}_{\mathbf{j}^{\prime} \sigma}^{\dagger} \tilde{c}_{\mathbf{i} \bar{\sigma}}^{\dagger} \tilde{c}_{\mathbf{i} \sigma} \tilde{c}_{\mathbf{j} \bar{\sigma}}\right) .
$$

We note that although this equation is written incorrectly in the article, the calculations discussed in Phys. Rev. B 92, 075119 (2015) follow the above-written (i.e., the correct) formula.

We thank M. M. Maśka for notifying us about the above typo. 